\title{
Spurensuche im Mikrokosmos
}

\section{Bruno Kesseli}

Dr. med. et lic. phil., Chefredaktor

Ist das nun ein Film darüber, was eine Depression ist? Oder eher über Neurobiologie und Molekulargenetik? Oder gar über das in der modernen Naturwissenschaft dominierende Welt- und Menschenbild? Fragen, die beim Anschauen des Films Das dunkle Gen durchaus auftauchen können, sich vielleicht nachgerade aufdrängen. Sicher ist, dass im Zentrum des Dokumentarfilms von Mirjam Jakobs und Gerhard Schick ein Mensch steht, der zwei schwere depressive Episoden durchgemacht hat. Frank Schauder, Arzt und Neurologe, begibt sich auf eine Spurensuche nach dem Ursprung seiner Erkrankung. Entscheidende Antworten erhofft er sich von den neuesten Forschungsergebnissen zur Neurobiologie des Zentralnervensystems und vor allem zum «Buch des Lebens», das beim Menschen in jeder (kernhaltigen) Zelle in Form eines knapp zwei Meter langen DNA-Strangs aus 3,27 Milliarden Basenpaaren vorliegt: dem Genom.

Um es vorwegzunehmen: Der Film bietet auf diesen beiden Ebenen - Neurobiologie und Genetik -, die Frank Schauder fast magisch in ihren Bann ziehen, zwar eine Fülle spannenden Materials, für dessen Präsentation die Autoren teilweise faszinierende filmische Ausdrucksformen finden. In Bezug auf die Erklärung und Veranschaulichung dessen, was eine Depression ist, bleibt die Ausbeute indessen dürftig. Dass die Biochemie des Zentralnervensystems und geneti- sche Faktoren im Zusammenhang mit depressiven Erkrankungen eine Rolle spielen, ist unbestritten. Auch Frank Schauder wusste ohne Laboruntersuchungen, dass in seiner Familie depressive Erkrankungen ge-

"Wir sind keine Übermenschen. Wir sind nur Ingenieure, die ihren Job machen.»

häuft auftreten. Dass sich kein singuläres «dunkles Gen» eruieren lässt, ist nicht weiter verwunderlich. Depressionen dürften "multifaktoriell» bedingt, der Filmtitel eher dramaturgischen und Marketingüberlegungen geschuldet sein. Es ist zu erwarten, dass die Forschung früher oder später erklären kann, wie die Veranlagung zu depressiven Erkrankungen auf genetischer Ebene zustande kommt. Ob sich auf diese Weise und auf der Ebene der Synapsen und Neurotransmitter das komplexe Phänomen «Depression» umfassend verstehen lässt, ist eine andere Frage.

So bringt denn auch die Analyse seines persönlichen Genoms, die Frank Schauder bei einem spezialisierten Unternehmen in Auftrag gibt, alles andere als bahnbrechende Erkenntnisse. Das Risiko für bipolare affektive Störungen, das sie zutage fördert, ist sehr gering. Zum Risiko für eine "Major Depression", die in seinem Fall zutreffende Diagnose, konnten offenbar (noch) keine Aussagen gemacht werden. Die befragten

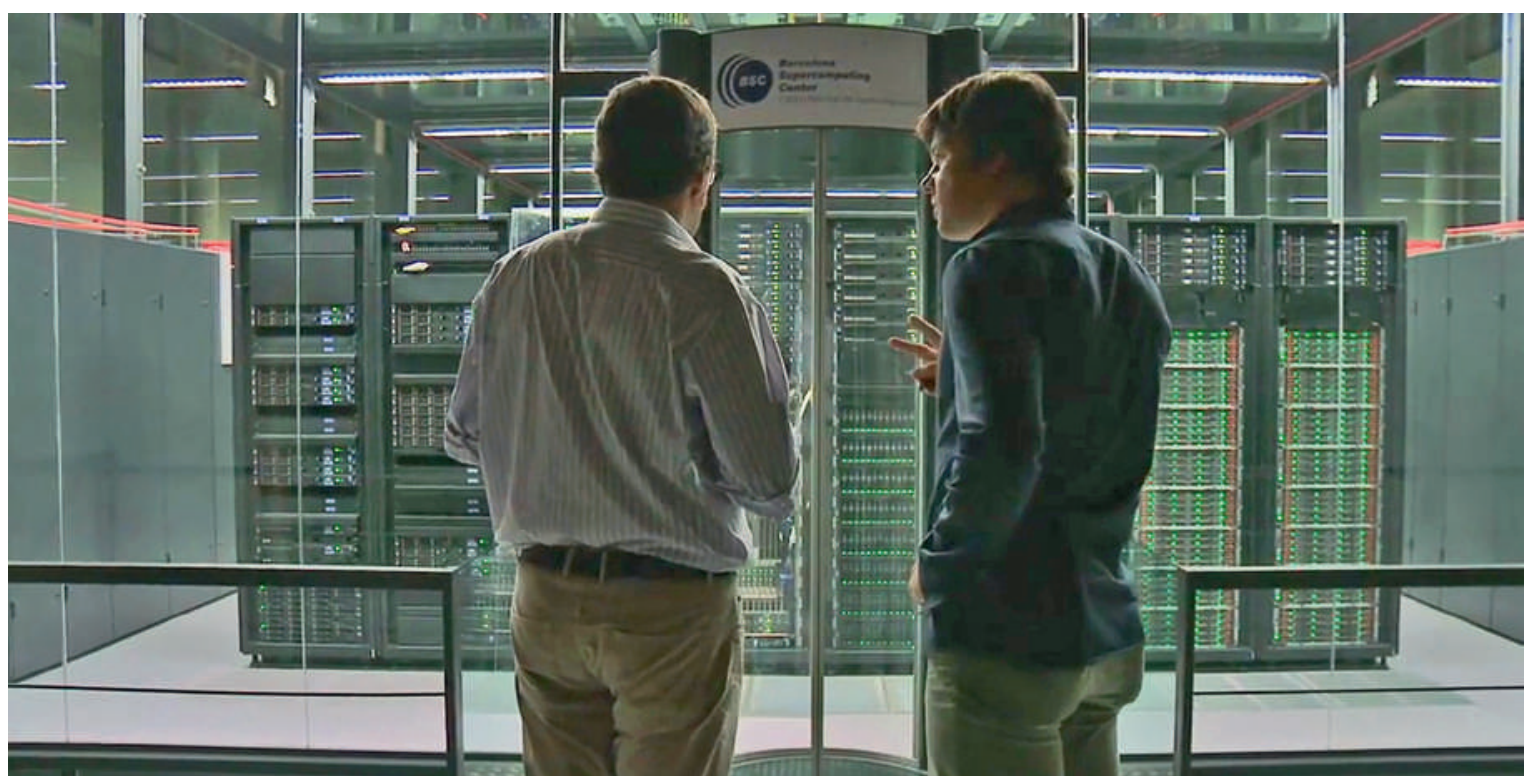

Allerheiligstes der Genanalyse: Frank Schauder (rechts) vor dem Supercomputer «MareNostrum» in Barcelona. 
Wissenschaftler bleiben in ihren Aussagen zu spezifischen Aspekten depressiver Erkrankungen ebenfalls vage.

Dagegen werden eindrückliche Einblicke in Grenzbereiche des modernen Wissenschaftsbetriebs und in die Denkwelten der an diesen Fronten tätigen Wissenschaftler vermittelt. "Wir sind gerade dabei, unser Menschsein zu verändern", sagt seelenruhig der Molekulargenetiker Georg Church, der fast so aussieht, wie man sich als Kleinkind den lieben Gott vorstellt. «Aber wir sind keine Übermenschen. Wir sind nur Ingenieure, die ihren Job machen, wie Ingenieure dies immer getan haben.» Der Supercomputer "MareNostrum» des Nationalen Genom-Analyse-Zentrums in Barcelona, das vom Schweizer Ivo Gut geleitet wird, steht bezeichnenderweise in einer ehemaligen Kathedrale.

Auch wenn man sich mitunter fragt, ob der Protagonist die Antworten auf seine Fragen zur Depression am richtigen Ort sucht, ist seine filmische Reise in vielerlei
Hinsicht bereichernd. Zu dieser Gesamtbilanz tragen auch die Bilder bei, die die Filmemacher finden, um die komplexen biochemischen und molekularbiologischen Vorgänge im Gehirn zu visualisieren, und die eine eigenartige, mitunter fast mystische Stimmung erzeugen. Dass sie die Pfade des Wissenschaftsbetriebs verschiedentlich verlassen und Künstler aufspüren, die Erkenntnisse aus der Molekularbiologie und Genetik in Musik oder Plastiken transformieren, erweitert den Film um eine wesentliche Dimension.

Die intensivsten Momente entstehen aber interessanterweise, wenn Frank Schauder ganz konventionell vor der Kamera davon erzählt, wie er seine Depression erlebt hat. In diesen Einstellungen sind sein Schmerz, seine Verzweiflung, seine Verlassenheit unmittelbar spürbar und man vermeint als Zuschauer, eine Ahnung davon zu erhalten, was es bedeutet, in einer Depression gefangen zu sein.

Das dunkle Gen läuft ab 28. Mai in den Schweizer Kinos.

\section{Wie Transgender-Patienten betreuen?}

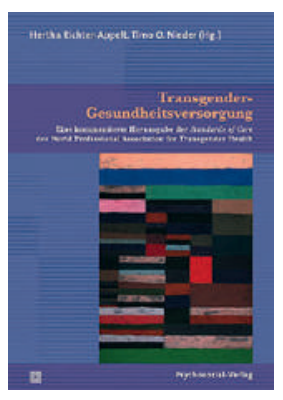

Hertha Richter-Appelt, Timo O. Nieder (Hg.)

Transgender-Gesundheitsversorgung

Eine kommentierte Herausgabe der Standards of Care der World Professional Association for Transgender Health

Giessen: Psychosozial-Verlag; 2014.

203 Seiten. 24.90 EUR

ISBN 978-3-8379-2424-4

Seit 1979 gibt die interdisziplinäre World Professional Association for Transgender Health (WPATH) Empfehlungen zur Betreuung von Menschen heraus, die an einer fehlenden Übereinstimmung ihrer subjektiven Geschlechtszugehörigkeit mit ihren geschlechtsspezifischen körperlichen Merkmalen leiden. Hertha Richter-Appelt und Timo O. Niederer, zwei anerkannte Spezialisten auf diesem Gebiet, haben die 7. Version der Vorsorgeempfehlungen aus dem Jahr 2011 aus dem Englischen übersetzt und kommentiert.

Erklärtes Ziel ist, den Patienten zu einer Linderung ihres Leidensdrucks, zu Zufriedenheit mit ihrer Identität und zu optimaler Selbstverwirklichung zu verhelfen. In diesem Zusammenhang wird gefordert, allen Patienten den Zugang zu entsprechenden psychischen, hormonellen und operativen Behandlungsmassnahmen zu ermöglichen. Gleichzeitig soll für keine der genannten Therapien ein Zwang bestehen; vielmehr soll ein individuell den Bedürfnissen des Patienten angepasster Behandlungsplan erarbeitet werden. Die einzelnen Behandlungsmethoden mit Zulassungskriterien und Kontraindikationen werden übersichtlich diskutiert.

Die Ausführungen beziehen sich auf den westlichen Kulturkreis. Allerdings stösst die Übertragbarkeit auf schweizerische Verhältnisse an Grenzen, insbesondere was den Vorschlag des Einfrierens von Eizellen betrifft, um nach einer Frau-zu-Mann-Operation nachträglich Nachkommen mittels Leihmutterschaft zu ermöglichen. Und bezüglich der Bedürfnisse von intersexuellen Menschen, deren Genitale bei Geburt uneindeutig ist oder deren körperliche sexuelle Entwicklung atypisch verläuft, wird auf separate Guidelines verwiesen. Für diejenigen Ärzte aber, die Patienten mit Transsexualität und Genderdysphorie betreuen, handelt es sich um ein gut strukturiertes, einfach lesbares und mit 203 Seiten überschaubares Werk.

Erika Nussberger, Vevey 